Journal of the Operations Research

Society of Japan

2008, Vol. 51, No. 3, 225-240

\title{
OPTIMAL TIME TO INVEST UNDER UNCERTAINTY WITH A SCALE CHANGE
}

\author{
Naoki Makimoto \\ University of Tsukuba
}

(Received November 7, 2006; Revised April 4, 2008)

\begin{abstract}
In this paper, we investigate the optimal investment problem to maximize expected discounted payoff of a project whose value is given by a product of two processes: a geometric Brownian motion representing continuous fluctuation over time and a Markov process which gives a discontinuous scale change. It turns out that the optimal policy is of threshold type whose thresholds depend on the current state of the Markov process. For 2-state case, the problem can be solved explicitly by using Bellman equation and smooth pasting conditions. On the other hand, the problem becomes much involved when there are multiple states. We exploit the structure of the optimal policy and the form of the value functions which enables us to develop a simple numerical procedure for computing the optimal policy and the value functions.
\end{abstract}

Keywords: Finance, optimal investment, stopping problem, Bellman equation, smooth pasting

\section{Introduction}

Recently, many papers have studied optimal investment problems under uncertainty. The main objective of these researches is to find optimal time to invest so as to maximize the expected future payoff under the assumptions on the stochastic dynamics of the project value, investment opportunity, investment cost, and so on. In contrast to the traditional approaches for valuing projects, these analyses makes it possible to explicitly incorporating such flexibilities as option to invest, defer and expansion into decision making. As a result, a variety of models have been analysed and applied to practical problems in the context of real option analysis [12].

The basic form of the optimal investment problem is to maximize expected discounted payoff of the project with a constant investment cost. Assuming that the project value follows a geometric Brownian motion, the optimal policy is shown to be of threshold type and the value function and the optimal threshold are explicitly obtained. Since the seminal book by Dixit and Pindyck [3], this basic problem has been extended in many directions. Décamps et al. [2] analyses a problem in which an investor has to choose among two alternative projects with different characteristics. Guo [5] and Guo et al. [6] introduce Markovian regime shifts into the drift and volatility of a geometric Brownian motion. Ohnishi and Tsujimura [10] considers an optimal investment problem with transaction costs. Vollert [13] discusses several types of optimal investment problem in a stochastic control framework.

In this paper, we investigate the optimal investment problem in which the project value is given by a product of two processes. One process follows a geometric Brownian motion which represents continuous fluctuation over time as in the most previous studies. Another source of uncertainty is given by a Markov process which gives a discontinuous scale change 
to the project value. From practical point of view, such a scale change will be caused, for example, by preemption or exit of other companies, drastic changes of product price, and so on. We first show that the optimal policy is of threshold type whose thresholds depend on the current state of the Markov process. By using the standard approach based on Bellman equation and smooth pasting conditions, the optimal solutions can be obtained explicitly for 2 -state case. On the other hand, the structure of the optimal policy becomes much involved when there are multiple states. We therefore exploit the structure of the optimal policy and the form of the value functions which enables us to develop a simple numerical procedure for computing the optimal policy and the value functions.

This paper is organized as follows. In Section 2, we formulate the optimal investment problem. In Section 3, we investigate the characteristics of the optimal policy. Section 4 is devoted to solving Bellman equation to identify the form of the value functions. Finally in Section 5, we consider some computational issues and show some numerical examples.

\section{The Optimal Investment Problem and Preliminary Results}

In this section, we describe an investment decision problem and formulate it as an optimization problem. Suppose there is a project to which we are going to invest some amount of money. At any time point until investment has been executed, we can choose either to invest now or to wait for future investment. If we decide to invest at $t$, then we will receive payoff $V_{t}-I$ where $V_{t}$ is a value of the project and $I$ is an investment cost.

In this paper, we assume that the project value is given by $V_{t}=S_{t} U_{t}$. Conceptually, $U_{t}$ represents the continuous fluctuation over time and $S_{t}$ gives a discontinuous scale change or multipilcative shock to the project value. As in standard real option models, we assume that $\left\{U_{t}\right\}$ follows a geometric Brownian motion:

$$
\frac{d U_{t}}{U_{t}}=\mu d t+\sigma d z_{t}
$$

where drift $\mu$ and volatility $\sigma$ are constant and $\left\{z_{t}\right\}$ is a standard Brownian motion. It is well known that the solution of Equation (2.1) is given as a geometric Brownian motion:

$$
U_{t}=U_{0} \exp \left\{\left(\mu-\sigma^{2} / 2\right) t+\sigma z_{t}\right\}
$$

On the other hand, $\left\{S_{t}\right\}$ is a continuous-time Markov process on the state space $\mathcal{S}=$ $\left\{s_{1}, \ldots, s_{N}\right\}$ governed by the transition rate matrix $\boldsymbol{Q}$. Throughout the paper, we assume without loss of generality $0<s_{1}<s_{2}<\cdots<s_{N}<\infty$. We also assume that $\left\{U_{t}\right\}$ and $\left\{S_{t}\right\}$ are mutually independent. A constant investment cost $I$ is incurred at the time of investment and a future payoff is discounted by an exogenously given discount rate $\rho$, as in the most literatures on real option analysis. To assure the existence of the expectation, we assume $\rho>\mu$.

The investment decision problem can be formulated as

$$
F_{i}(u)=\sup _{\tau \geq 0} E\left[e^{-\rho \tau}\left(S_{\tau} U_{\tau}-I\right) \mid S_{0}=s_{i}, U_{0}=u\right]=\sup _{\tau \geq 0} E_{i, u}\left[e^{-\rho \tau}\left(S_{\tau} U_{\tau}-I\right)\right] .
$$

The supremum is taken over all stopping times with respect to the filtration generated by $\left\{U_{t}\right\}$ and $\left\{S_{t}\right\}$. Here and in what follows, we use the abbreviated notation $E_{i, u}[\cdot]$ instead of $E\left[\cdot \mid S_{0}=s_{i}, U_{0}=u\right]$ for notational simplicity.

For later use, we introduce the optimal solution when $S_{t}=s$ is a constant. The problem in this case is to obtain

$$
H(u ; s)=\sup _{\tau \geq 0} E\left[e^{-\rho \tau}\left(s U_{\tau}-I\right) \mid U_{0}=u\right]
$$


(we specify the dependence of $H(u ; s)$ on $s$ ). Then, as described in Dixit and Pindyck [3], the optimal policy is to invest at the first time $U_{t} \geq v$ occurs and the value function under this policy is given by

$$
H(u ; s)= \begin{cases}\left(\frac{s v-I}{v^{\xi}}\right) u^{\xi}, & 0<u<v \\ s u-I, & u \geq v\end{cases}
$$

where

$$
v=\frac{\xi I}{(\xi-1) s}, \quad \xi=f^{+}(\rho)>1
$$

In the sequel, we use the notations

$$
\begin{aligned}
& f^{+}(a)=\frac{\sigma^{2} / 2-\mu+\sqrt{\left(\sigma^{2} / 2-\mu\right)^{2}+2 \sigma^{2} a}}{\sigma^{2}} \quad(>1, \forall a>0), \\
& f^{-}(a)=\frac{\sigma^{2} / 2-\mu-\sqrt{\left(\sigma^{2} / 2-\mu\right)^{2}+2 \sigma^{2} a}}{\sigma^{2}} \quad(<0, \forall a>0)
\end{aligned}
$$

to represent the solutions of a quadratic equation

$$
\frac{\sigma^{2}}{2} x^{2}-\left(\frac{\sigma^{2}}{2}-\mu\right) x-a=0
$$

Remark 2.1 In real option analysis, it is common to define the objective function (2.3) by

$$
\sup _{\tau \geq 0} E_{i, u}\left[e^{-\rho \tau} \max \left(S_{\tau} U_{\tau}-I, 0\right)\right]
$$

to clarify the analogy with financial call options [3]. It is however obvious that Equations (2.3) and (2.8) lead to the same value function and optimal investment time since it is never optimal to invest when $S_{t} U_{t}<I$ and, starting with any initial values of $S_{0} \in \mathcal{S}$ and $U_{0}>0$, $S_{t} U_{t}>I$ occurs at some future time $t$ with probability 1 .

Before closing this section, we state the following result whose proof can be found in a standard textbook on differential equations.

Lemma 2.1 For $a \geq 0$, the solution of the ordinary differential equation

$$
-a g(u)+\mu u g^{\prime}(u)+\frac{\sigma^{2}}{2} u^{2} g^{\prime \prime}(u)+b u+c=0
$$

is given by

$$
g(u)=d_{1} u^{\xi_{1}}+d_{2} u^{\xi_{2}}+\frac{b}{a-\mu} u+\frac{c}{a}
$$

where

$$
\xi_{1}=f^{+}(a)>1, \quad \xi_{2}=f^{-}(a)<0 .
$$

Coefficients $d_{1}, d_{2}$ should be determined by boundary conditions. 


\section{Characterization of the Optimal Policy}

In this section, we investigate the characteristics of the optimal investment policy. The next lemma indicates that the optimality of threshold policy for the problem (2.4) is inherited even when $S_{t}$ is stochastic. Optimal policy of threshold type can be found in a variety of investment problems $[2,3,5,6,10,13]$.

Lemma 3.1 The optimal investment policy is of threshold type, i.e., corresponding to the state of $S_{t}$, there exists a threshold $v_{i}(i=1, \ldots, N)$ such that it is optimal to invest at time $t$ if $S_{t}=s_{i}$ and $U_{t} \geq v_{i}$, and it is optimal to continue waiting otherwise.

(Proof) Fix $S_{0}=s_{i}$. Suppose to the contrary that there exist $u<\tilde{u}$ such that it is optimal to invest when $U_{0}=u$ while waiting is optimal when $U_{0}=\tilde{u}$. From Equation (2.2), we can define $U_{t}=u \exp \left\{\left(\mu-\sigma^{2} / 2\right) t+\sigma z_{t}\right\}$ and $\tilde{U}_{t}=\tilde{u} \exp \left\{\left(\mu-\sigma^{2} / 2\right) t+\sigma z_{t}\right\}$ by the same Brownian motion $\left\{z_{t}\right\}$. From the assumption, we have

$$
s_{i} u-I \geq E_{i, u}\left[e^{-\rho \tau}\left(S_{\tau} U_{\tau}-I\right)\right]
$$

for any $\left\{\mathcal{F}_{t}\right\}$-stopping time $\tau$, and

$$
s_{i} \tilde{u}-I<E_{i, \tilde{u}}\left[e^{-\rho \tilde{\tau}}\left(S_{\tilde{\tau}} \tilde{U}_{\tilde{\tau}}-I\right)\right]
$$

where $\tilde{\tau}$ is the optimal stopping time for $\left\{\tilde{U}_{t}\right\}$. If one uses the policy to invest at $\tilde{\tau}$ for $\left\{U_{t}\right\}$, we obtain from $u<\tilde{u}$ that

$$
e^{-\rho \tilde{\tau}}\left(S_{\tilde{\tau}} U_{\tilde{\tau}}-I\right)=e^{-\rho \tilde{\tau}}\left(\frac{u}{\tilde{u}} S_{\tilde{\tau}} \tilde{U}_{\tilde{\tau}}-I\right)>\frac{u}{\tilde{u}} e^{-\rho \tilde{\tau}}\left(S_{\tilde{\tau}} \tilde{U}_{\tilde{\tau}}-I\right)-\left(1-\frac{u}{\tilde{u}}\right) I .
$$

By taking the expectation on both sides of Equation (3.3) and using Equation (3.2), we get

$$
\begin{aligned}
E_{i, u}\left[e^{-\rho \tilde{\tau}}\left(S_{\tilde{\tau}} U_{\tilde{\tau}}-I\right)\right] & >\frac{u}{\tilde{u}} E_{i, \tilde{u}}\left[e^{-\rho \tilde{\tau}}\left(S_{\tilde{\tau}} \tilde{U}_{\tilde{\tau}}-I\right)\right]-\left(1-\frac{u}{\tilde{u}}\right) I \\
& >\frac{u}{\tilde{u}}\left(s_{i} \tilde{u}-I\right)-\left(1-\frac{u}{\tilde{u}}\right) I \\
& =s_{i} u-I .
\end{aligned}
$$

Since the inequality (3.4) contradicts the fact that (3.1) holds for any $\tau$, this completes the proof.

Since the project value $V_{t}=S_{t} U_{t}$ changes discontinuously when $S_{t}$ transits from one state to another, an investment will be executed at the time either $U_{t}$ reaches the threshold $v_{i}$ from below while $S_{t}=s_{i}$ remains unchanged, or $S_{t}$ transits from $s_{i}$ to $s_{i+1}$ when $v_{i+1} \leq U_{t}<v_{i}$.

From Lemma 3.1, we see

$$
\begin{array}{ll}
F_{i}(u)>s_{i} u-I, & 0<u<v_{i}, \\
F_{i}(u)=s_{i} u-I, & u \geq v_{i} .
\end{array}
$$

If $v_{i}<\infty, F_{i}(u)$ connects to the straight line $s_{i} u-I$ from above at $u=v_{i}$. It should be noted that some of $v_{i}$ may be infinite which means it is never optimal to invest when $S_{t}=s_{i}$. However, at least $v_{N}<\infty$ as shown in the next lemma.

Lemma $3.2 v_{N}<v_{1}$ and $v_{N}<\infty$.

(Proof) If $s_{1}=\cdots=s_{N}=s$, all value functions must equal $H(u ; s)$. Since $s_{1}<s_{2}<\cdots<$ $s_{N}$, we therefore obtain

$$
H\left(u ; s_{1}\right) \leq F_{1}(u), \quad F_{N}(u) \leq H\left(u ; s_{N}\right), \quad \forall u>0
$$


Notice that the graph of $H(u ; s)$ connects from above to the straight line $s u-I$ at $u=\frac{\xi I}{(\xi-1) s}$ [3]. Since $F_{i}(u) \geq s_{i} u-I$ for all $u>0$, it is clear that $\frac{\xi I}{(\xi-1) s_{1}} \leq v_{1}$ and $v_{N} \leq \frac{\xi I}{(\xi-1) s_{N}}$ must hold so as to satisfy (3.5). The result follows since $\frac{\xi I}{(\xi-1) s_{N}}<\frac{\xi I}{(\xi-1) s_{1}}<\infty$.

When $\left\{S_{t}\right\}$ has only two states, we see $v_{2}<v_{1}$ from Lemma 3.2. However, the order of $v_{i}$ 's is not automatically determined when there are more than two states. In general, there seems to exist no simple way to identify the order of $v_{i}$ 's. This makes the problem of solving Bellman equation to obtain the value functions much involved.

In what follows, we provide a simple and useful condition to assure $v_{N} \leq \cdots \leq v_{1}$. Let $S_{t}^{(i)}$ denote the conditional process of $S_{t}$ starting with $S_{0}=s_{i}$.

Theorem 3.1 If one can construct sample paths of $\left\{S_{t}^{(i)}\right\}$ and $\left\{S_{t}^{(i+1)}\right\}$ on the same probability space so as to satisfy

$$
P\left(S_{t}^{(i+1)}-S_{t}^{(i)} \leq s_{i+1}-s_{i}, \forall t \geq 0\right)=1,
$$

then $v_{i+1} \leq v_{i}$.

The meaning of the condition in Theorem 3.1 seems unclear at first sight. Before proving the theorem, we give an illustrative example which satisfies the condition.

Example 3.1 Suppose that $\left\{S_{t}\right\}$ follows a birth-death process with the transition rate matrix

$$
\boldsymbol{Q}=\left(\begin{array}{ccccc}
-\alpha_{1} & \alpha_{1} & & & \boldsymbol{O} \\
\beta_{2} & -\left(\alpha_{2}+\beta_{2}\right) & \ddots & & \\
& \ddots & \ddots & \ddots & \\
\boldsymbol{O} & & \ddots & -\left(\alpha_{N-1}+\beta_{N-1}\right) & \alpha_{N-1} \\
& & & \beta_{N} & -\beta_{N}
\end{array}\right)
$$

and the state space is composed of $N$ equally spaced states with unit interval $s$, i.e., $s_{k}=$ $s_{1}+(k-1) s$ for $k=2, \ldots, N$. If the transition rates satisfy

$$
\alpha_{1} \geq \alpha_{2} \geq \cdots \geq \alpha_{N-1}, \quad \beta_{2} \leq \beta_{3} \leq \cdots \leq \beta_{N},
$$

then the condition in Theorem 3.1 is satisfied for all $i=1, \ldots, N-1$ as we will see below.

The condition (3.7) implies that the lower (higher, respectively) the current state is, the more likely the process will move to higher (lower) state. This property is so called mean reversion which is observed in a variety of financial time series such as interest rate and price of commodities.

To see that the condition (3.7) implies that in Theorem 3.1 , let $\boldsymbol{P}=\boldsymbol{I}+\boldsymbol{Q} / \theta$ be a transition probability matrix obtained by uniformization where $\theta=\max _{1 \leq k \leq N}\left\{\alpha_{k}+\beta_{k}\right\}$. Denote by $\left\{\bar{S}_{n}\right\}$ a discrete time Markov chain governed by $\boldsymbol{P}$ and let $\{N(t)\}$ be a Poisson process with intensity $\theta$ which is independent of $\left\{\bar{S}_{n}\right\}$ and $\left\{z_{t}\right\}_{t>0}$. The subordinated process $S_{t}=\bar{S}_{N(t)}$ is a continuous time Markov chain governed by $\boldsymbol{Q}$ (see Example 4.2 of Kijima [7]). Let $Y_{1}, Y_{2}, \ldots$ be a sequence of independent uniform random variables which is independent of $\{N(t)\}$. We construct the conditional process $\left\{\bar{S}_{n}^{(j)}\right\}$ starting with $s_{j}$ by setting $\bar{S}_{0}^{(j)}=s_{j}$ and

$$
\bar{S}_{n+1}^{(j)}= \begin{cases}s_{k}-s, & Y_{n+1}<\beta_{k} / \theta, \\ s_{k}, & \beta_{k} / \theta \leq Y_{n+1}<1-\alpha_{k} / \theta, \\ s_{k}+s, & 1-\alpha_{k} / \theta \leq Y_{n+1}\end{cases}
$$

when $\bar{S}_{n}^{(j)}=s_{k}$. Here we use the convention $\alpha_{N}=\beta_{1}=0$. If we generate sample paths of $\left\{\bar{S}_{n}^{(i)}\right\}$ and $\left\{\bar{S}_{n}^{(i+1)}\right\}$ using common $\left\{Y_{n}\right\}$, it can be readily checked from (3.8) by induction 
that $\bar{S}_{n}^{(i+1)}-\bar{S}_{n}^{(i)} \leq s_{i+1}-s_{i}, \forall n=0,1, \ldots$ By setting $S_{t}^{(j)}=\bar{S}_{N(t)}^{(j)}$ for $j=i, i+1$, we can construct sample paths of $\left\{S_{t}^{(i)}\right\}$ and $\left\{S_{t}^{(i+1)}\right\}$ which satisfy Equation (3.6).

Now we prove Theorem 3.1.

(Proof of Theorem 3.1) Suppose to the contrary that $v_{i}<v_{i+1}$ and choose arbitrary $u \in$ $\left(v_{i}, v_{i+1}\right)$. Let $\tilde{\tau}$ be the optimal investment time starting with $S_{0}=s_{i+1}$ and $U_{0}=u$. From the choices of $u$ and $\tilde{\tau}$, we have

$$
\begin{aligned}
F_{i}(u) & =s_{i} u-I>E_{i, u}\left[e^{-\rho \tilde{\tau}}\left(S_{\tilde{\tau}}^{(i)} U_{\tilde{\tau}}-I\right)\right], \\
F_{i+1}(u) & =E_{i+1, u}\left[e^{-\rho \tilde{\tau}}\left(S_{\tilde{\tau}}^{(i+1)} U_{\tilde{\tau}}-I\right)\right]>s_{i+1} u-I .
\end{aligned}
$$

By the assumption of Theorem 3.1, we can obtain

$$
e^{-\rho \tilde{\tau}}\left(S_{\tilde{\tau}}^{(i+1)} U_{\tilde{\tau}}-I\right)-e^{-\rho \tilde{\tau}}\left(S_{\tilde{\tau}}^{(i)} U_{\tilde{\tau}}-I\right) \leq e^{-\rho \tilde{\tau}}\left(s_{i+1}-s_{i}\right) U_{\tilde{\tau}}
$$

for any sample path of $\left\{U_{t}\right\}$ independently chosen from $\left\{S_{t}^{(j)}\right\}(j=i, i+1)$ and for resulting sample of $\tilde{\tau}$ determined by $\left\{S_{t}^{(i+1)}\right\}$ and $\left\{U_{t}\right\}$. Then,

$$
\begin{aligned}
F_{i+1}(u) & =E_{i+1, u}\left[e^{-\rho \tilde{\tau}}\left(S_{\tilde{\tau}}^{(i+1)} U_{\tilde{\tau}}-I\right)\right] \\
& \leq E_{i, u}\left[e^{-\rho \tilde{\tau}}\left(S_{\tilde{\tau}}^{(i)} U_{\tilde{\tau}}-I\right)\right]+\left(s_{i+1}-s_{i}\right) E_{i+1, u}\left[e^{-\rho \tilde{\tau}} U_{\tilde{\tau}}\right] \\
& <s_{i} u-I+\left(s_{i+1}-s_{i}\right) u \\
& =s_{i+1} u-I .
\end{aligned}
$$

Inequality (3.12) follows by taking expectation of (3.11) with respect to the independent probability measures on $\left\{S_{t}^{(j)}\right\}$ and $\left\{U_{t}\right\}$. To show inequality (3.13), note that

$$
u \geq E_{i+1, u}\left[e^{-\rho \min (\tilde{\tau}, T)} U_{\min (\tilde{\tau}, T)}\right] \geq E_{i+1, u}\left[e^{-\rho \tilde{\tau}} U_{\tilde{\tau}} 1_{\{\tilde{\tau} \leq T\}}\right]
$$

holds for all $T>0$ where $1_{A}$ is an indicator function of $A$. The first inequality in (3.15) follows from optional sampling theorem applied to supermartingale $\left\{e^{-\rho t} U_{t}\right\}$ and the second one follows from $U_{t}>0$. Since $P(\tilde{\tau}<\infty)=1$, we obtain from monotone convergence theorem

$$
\lim _{T \rightarrow \infty} E_{i+1, u}\left[e^{-\rho \tilde{\tau}} U_{\tilde{\tau}} 1_{\{\tilde{\tau} \leq T\}}\right]=E_{i+1, u}\left[e^{-\rho \tilde{\tau}} U_{\tilde{\tau}}\right]
$$

which together with inequalities (3.9) and (3.15) prove (3.13). Since inequality (3.14) contradicts $(3.10)$, the proof is completed.

In addition to the order of $v_{i}$ 's, we need to know whether $v_{i}<\infty$ or not to solve Bellman equation. To this end, let $\mathcal{S}_{k}=\left\{s_{k+1}, s_{k+2}, \ldots, s_{N}\right\}$ and let $\kappa_{i k}$ be the first passage time of $S_{\mathrm{t}}$ from $s_{i}$ into $\mathcal{S}_{k}$. Now suppose $S_{0}=s_{i}$ and $U_{0}=u$ and consider the following policy:

(a) invest at $\kappa_{i k}$ irrespective of the value of $U_{\kappa_{i k}}$.

Denote by $G_{i k}(u)$ the expected payoff of policy (a). Since $\left\{S_{t}\right\}$ and $\left\{U_{t}\right\}$ are assumed to be independent, $G_{i k}(u)$ is calculated as

$$
\begin{aligned}
G_{i k}(u) & =\sum_{j=k+1}^{N} E_{i, u}\left[e^{-\rho \kappa_{i k}}\left(s_{j} U_{\kappa_{i k}}-I\right) 1_{\left\{S_{\kappa_{i k}}=s_{j}\right\}}\right] \\
& =\sum_{j=k+1}^{N} \int_{0}^{\infty} e^{-\rho t} E_{i, u}\left[s_{j} U_{t}-I\right] P\left(\kappa_{i k} \leq d t, S_{t}=s_{j}\right) \\
& =\sum_{j=k+1}^{N} \int_{0}^{\infty} e^{-\rho t}\left(s_{j} u e^{\mu t}-I\right) P\left(\kappa_{i k} \leq d t, S_{t}=s_{j}\right) \\
& =\sum_{j=k+1}^{N}\left[f_{i j}^{(k)}(\rho-\mu) s_{j} u-f_{i j}^{(k)}(\rho) I\right]
\end{aligned}
$$


where

$$
f_{i j}^{(k)}(s)=\int_{0}^{\infty} e^{-s t} P\left(\kappa_{i k} \leq d t, S_{t}=s_{j}\right)=E\left[e^{-s \kappa_{i k}} 1_{\left\{S_{\kappa_{i k}}=s_{j}\right\}}\right] .
$$

$f_{i j}^{(k)}(s)$ can be calculated as follows. Let $\boldsymbol{Q}_{1}^{(k)}$ and $\boldsymbol{Q}_{2}^{(k)}$ respectively be $k \times k$ north-west corner and $k \times(N-k)$ north-east corner truncations of the transition rate matrix $\boldsymbol{Q}$. For $i=1, \ldots, k$ and $j=k+1, \ldots, N$, we obtain

$$
f_{i j}^{(k)}(s)=\left[\int_{0}^{\infty} e^{-s t} \exp \left\{\boldsymbol{Q}_{1}^{(k)} t\right\} \boldsymbol{Q}_{2}^{(k)} d t\right]_{i, j-k}=\left[\left\{s \boldsymbol{I}-\boldsymbol{Q}_{1}^{(k)}\right\}^{-1} \boldsymbol{Q}_{2}^{(k)}\right]_{i, j-k}
$$

where $\boldsymbol{I}$ is an identity matrix and $[\boldsymbol{A}]_{i j}$ denotes $(i, j)$ th component of matrix $\boldsymbol{A}$, cf., [7].

The next lemma characterizes asymptotic of $F_{i}(u)$ as $u \rightarrow \infty$ if $v_{i}=\infty$. For two functions $g(u)$ and $h(u)$, we write $g(u) \approx h(u)$ if $\lim _{u \rightarrow \infty}\{g(u)-h(u)\}=0$.

Lemma 3.3 Assume $v_{i}=\infty$ for $i=1, \ldots, k$ and $v_{j}<\infty$ for $j=k+1, \ldots, N$. Then, $F_{i}(u) \approx G_{i k}(u)$ for $i=1, \ldots, k$.

(Proof) Since $F_{i}(u)$ is the optimal value function, $F_{i}(u) \geq G_{i k}(u)$ for all $u>0$. The opposite inequality is derived from

$$
\begin{aligned}
F_{i}(u)-G_{i k}(u) & =\sum_{j=k+1}^{N} E_{i, u}\left[e^{-\rho \kappa_{i k}}\left\{F_{j}\left(U_{\kappa_{i k}}\right)-\left(s_{j} U_{\kappa_{i k}}-I\right)\right\} 1_{\left\{S_{\kappa_{i k}}=s_{j}, U_{\kappa_{i k}}<v_{j}\right\}}\right] \\
& \leq \sum_{j=k+1}^{N} E_{i, u}\left[e^{-\rho \kappa_{i k}}\left\{\left(s_{j} v_{j}-I\right)-\left(s_{j} U_{\kappa_{i k}}-I\right)\right\} 1_{\left\{S_{\kappa_{i k}}=s_{j}, U_{\kappa_{i k}}<v_{j}\right\}}\right] \\
& \leq \sum_{j=k+1}^{N} s_{j} v_{j} E_{i, u}\left[1_{\left\{S_{\kappa_{i k}}=s_{j}, U_{\kappa_{i k}}<v_{j}\right\}}\right] \\
& \leq \sum_{j=k+1}^{N} s_{j} v_{j} \int_{0}^{\infty} P\left(U_{t}<v_{j} \mid U_{0}=u\right) P\left(\kappa_{i k} \leq d t, S_{t}=s_{j}\right) .
\end{aligned}
$$

Since $P\left(U_{t}<v_{j} \mid U_{0}=u\right)$ monotonically decreases to 0 as $u \rightarrow \infty, \lim _{u \rightarrow \infty}\left\{F_{i}(u)-G_{i k}(u)\right\} \leq$ 0 by monotone convergence theorem.

The next theorem gives a complete answer to the question whether $v_{i}<\infty$ or not provided that $v_{N} \leq \cdots \leq v_{1}$. Let $g_{i}^{(k)}(s)=\sum_{j=k+1}^{N} f_{i j}^{(k)}(s) s_{j}$ and $h_{i}^{(k)}(s)=\sum_{j=k+1}^{N} f_{i j}^{(k)}(s)$ so that $G_{i k}(u)=g_{i}^{(k)}(\rho-\mu) u-h_{i}^{(k)}(\rho) I$.

Theorem 3.2 Assume $v_{N} \leq \cdots \leq v_{1}$. Let

$$
k^{*}=\max \left\{k=1, \ldots, N-1: s_{k} \leq g_{k}^{(k)}(\rho-\mu)\right\}
$$

with the understanding that $k^{*}=0$ if $s_{k}>g_{k}^{(k)}(\rho-\mu)$ for all $k=1, \ldots, N-1$. Then $v_{k}=\infty$ for $k=1, \ldots, k^{*}$ and $v_{k}<\infty$ for $k=k^{*}+1, \ldots, N$.

(Proof) Since $s_{k^{*}} \leq g_{k^{*}}^{\left(k^{*}\right)}(\rho-\mu)$ and $h_{k^{*}}^{\left(k^{*}\right)}(\rho)<1$, we obtain $s_{k^{*}} u-I<G_{k^{*} k^{*}}(u)$ for all $u>0$. This means that it is never optimal to invest when $S_{t}=s_{k^{*}}$ and $U_{t}=u$ for all $u>0$. Thus, $v_{k^{*}}=\infty$. Since $v_{k}$ is decreasing by assumption, $v_{k}=\infty$ for all $k=1, \ldots, k^{*}$. For $k=k^{*}+1, \ldots, N-1, s_{k}>g_{k}^{(k)}(\rho-\mu)$ and we obtain

$$
\lim _{u \rightarrow \infty}\left[F_{k}(u)-G_{k k}(u)\right] \geq \lim _{u \rightarrow \infty}\left[\left(s_{k} u-I\right)-\left\{g_{k}^{(k)}(\rho-\mu) u-h_{k}^{(k)}(\rho) I\right\}\right]=\infty .
$$

If there exists $k^{\prime} \in\left\{k^{*}+1, \ldots, N-1\right\}$ such that $v_{N} \leq \cdots \leq v_{k^{\prime}+1}<\infty$ and $v_{k^{\prime}}=\cdots=$ $v_{k^{*}+1}=\infty$, then $F_{k^{\prime}}(u) \approx G_{k^{\prime} k^{\prime}}(u)$ holds from Lemma 3.3. Since this is impossible from (3.21), no such $k^{\prime}$ exists and $v_{k}<\infty$ for all $k=k^{*}+1, \ldots, N$. 


\section{Bellman Equation and the Value Functions}

In this section, we solve Bellman equation to identify the form of the value functions. Throughout the section, we assume $v_{N}<\cdots<v_{k^{*}+1}<\infty$ and $v_{k^{*}}=\cdots=v_{1}=\infty$ for some $k^{*}=0, \ldots, N-1$.

Let $\lambda_{i}^{(k)}(i=1, \ldots, k)$ denote eigenvalue of $\boldsymbol{Q}_{1}^{(k)}$ and let $\boldsymbol{\ell}_{i}^{(k)}=\left(\ell_{i 1}^{(k)}, \ldots, \ell_{i k}^{(k)}\right)$ and $\boldsymbol{r}_{i}^{(k)}=$ $\left(r_{i 1}^{(k)}, \ldots, r_{i k}^{(k)}\right)^{\top}$ be associated left and right eigenvectors ( $\top$ denotes transpose): $\boldsymbol{\ell}_{i}^{(k)} \boldsymbol{Q}_{1}^{(k)}=$ $\lambda_{i}^{(k)} \boldsymbol{\ell}_{i}^{(k)}, \boldsymbol{Q}_{1}^{(k)} \boldsymbol{r}_{i}^{(k)}=\lambda_{i}^{(k)} \boldsymbol{r}_{i}^{(k)}$. Without loss of generality, we normalize $\boldsymbol{\ell}_{i}^{(k)}$ and $\boldsymbol{r}_{i}^{(k)}$ so as to satisfy $\boldsymbol{\ell}_{i}^{(k)} \boldsymbol{r}_{i}^{(k)}=1$. For simplicity, we assume that $\lambda_{1}^{(k)}, \ldots, \lambda_{k}^{(k)}$ are all distinct real numbers. This condition is fulfilled when $\left\{S_{t}\right\}$ is a birth-death process as in Example 3.1. It is also well known that the transition rate matrix of a time reversible Markov chain has real eigenvalues [7]. Define three $k \times k$ matrices by

$$
\boldsymbol{L}^{(k)}=\left(\begin{array}{c}
\boldsymbol{\ell}_{1}^{(k)} \\
\vdots \\
\boldsymbol{\ell}_{k}^{(k)}
\end{array}\right), \quad \boldsymbol{R}^{(k)}=\left(\boldsymbol{r}_{1}^{(k)}, \ldots, \boldsymbol{r}_{k}^{(k)}\right), \quad \boldsymbol{\Lambda}^{(k)}=\left(\begin{array}{ccc}
\lambda_{1}^{(k)} & & \boldsymbol{O} \\
& \ddots & \\
\boldsymbol{O} & & \lambda_{k}^{(k)}
\end{array}\right)
$$

which, by definition, satisfy

$$
\boldsymbol{L}^{(k)} \boldsymbol{Q}_{1}^{(k)}=\boldsymbol{\Lambda}^{(k)} \boldsymbol{L}^{(k)}, \quad \boldsymbol{Q}_{1}^{(k)} \boldsymbol{R}^{(k)}=\boldsymbol{\Lambda}^{(k)} \boldsymbol{R}^{(k)}, \quad \boldsymbol{L}^{(k)} \boldsymbol{R}^{(k)}=\boldsymbol{R}^{(k)} \boldsymbol{L}^{(k)}=\boldsymbol{I} .
$$

Let $\boldsymbol{F}^{(k)}(u)=\left(F_{1}(u), \cdots, F_{k}(u)\right)^{\top}$ and $\boldsymbol{H}^{(k)}=\boldsymbol{L}^{(k)} \boldsymbol{F}^{(k)}$. From Equation (4.1), an inversion relation $\boldsymbol{F}^{(k)}(u)=\boldsymbol{R}^{(k)} \boldsymbol{H}^{(k)}(u)$ also holds.

Define $\mathcal{I}_{N}=\left(0, v_{N}\right)$ and $\mathcal{I}_{k}=\left(v_{k+1}, v_{k}\right)$ for $k=N-1, \ldots, k^{*}$. From Lemma 3.1, $\mathcal{I}_{k}$ is a continuation region of $F_{i}(u)$ for $i=1, \ldots, k$, i.e., it is optimal to continue waiting if $S_{t}=s_{i}$ and $u \in \mathcal{I}_{k}$ and $F_{i}(u)>s_{i} u-I$ for $i=1, \ldots, k$. In what follows, we derive Bellman equation on $\mathcal{I}_{N}, \mathcal{I}_{N-1}, \ldots, \mathcal{I}_{k^{*}}$ in this order.

(i) $u \in \mathcal{I}_{N}$ : Since $u \in \mathcal{I}_{N}$ is a continuation region for all states of $S_{t}$, a system of Bellman equation is

$$
-\rho \boldsymbol{F}^{(N)}(u)+\mu u \boldsymbol{F}^{(N) \prime}(u)+\frac{\sigma^{2}}{2} u^{2} \boldsymbol{F}^{(N) \prime \prime}(u)+\boldsymbol{Q} \boldsymbol{F}^{(N)}(u)=\mathbf{0}, \quad u \in \mathcal{I}_{N}
$$

where $\boldsymbol{F}^{(N) \prime}(u)$ and $\boldsymbol{F}^{(N) \prime \prime}(u)$ are component-wise derivatives. The term $\boldsymbol{Q} \boldsymbol{F}^{(N)}(u)$ represents the effect caused by a state transition of $S_{t}$. See e.g., Fleming and Soner [4] and Vollert [13] for Bellman equation when the underlying process has a jump.

To transform the system of equations into independent equations, we pre-multiply Equation (4.2) by $\boldsymbol{L}^{(N)}$ to obtain

$$
-\left(\rho \boldsymbol{I}-\boldsymbol{\Lambda}^{(N)}\right) \boldsymbol{H}^{(N)}(u)+\mu u \boldsymbol{H}^{(N) \prime}(u)+\frac{\sigma^{2}}{2} u^{2} \boldsymbol{H}^{(N) \prime \prime}(u)=\mathbf{0}, \quad u \in \mathcal{I}_{N} .
$$

Since Equation (4.3) is a set of $N$ independent differential equations of the form of Equation (2.9), we can solve them explicitly from Lemma 2.1 as

$$
H_{i}^{(N)}(u)=a_{i}^{(N)} u^{l_{i}^{(N)}}+b_{i}^{(N)} u^{\xi_{i}^{(N)}}, \quad u \in \mathcal{I}_{N}
$$

where

$$
\eta_{i}^{(N)}=f^{+}\left(\rho-\lambda_{i}^{(N)}\right)>1, \quad \xi_{i}^{(N)}=f^{-}\left(\rho-\lambda_{i}^{(N)}\right)<0
$$


and $a_{i}^{(N)}, b_{i}^{(N)}$ are unknown coefficients. Note that since $\lim _{u \rightarrow 0} F_{i}(u)=0$ for all $i=1, \ldots, N$, we obtain $\lim _{u \rightarrow 0} H_{i}(u)=0$. In order to satisfy this boundary condition, the second term in Equation (4.4) must vanish and we have

$$
H_{i}^{(N)}(u)=a_{i}^{(N)} u^{\eta_{i}^{(N)}}, \quad u \in \mathcal{I}_{N}, \quad i=1, \ldots, N .
$$

(ii) $u \in \mathcal{I}_{k}\left(k=N-1, \ldots, k^{*}+1\right): \quad$ Since $\mathcal{I}_{k}$ is a continuation region of $F_{1}(u), \ldots, F_{k}(u)$ while $F_{i}(u)=s_{i} u-I$ for $i=k+1, \ldots, N$, a system of Bellman equation on $\mathcal{I}_{k}$ becomes

$$
-\rho \boldsymbol{F}^{(k)}(u)+\mu u \boldsymbol{F}^{(k) \prime}(u)+\frac{\sigma^{2}}{2} u^{2} \boldsymbol{F}^{(k) \prime \prime}(u)+\boldsymbol{Q}_{1}^{(k)} \boldsymbol{F}^{(k)}(u)+\boldsymbol{Q}_{2}^{(k)} \boldsymbol{g}^{(k)}(u)=\mathbf{0}, \quad u \in \mathcal{I}_{k}
$$

where $(N-k)$-dimensional column vector $\boldsymbol{g}^{(k)}(u)$ is given as

$$
\boldsymbol{g}^{(k)}(u)=\left(s_{k+1} u-I, s_{k+2} u-I, \ldots, s_{N} u-I\right)^{\top}
$$

Pre-multiplying Equation (4.6) by $\boldsymbol{L}^{(k)}$ yields

$$
-\left(\rho \boldsymbol{I}-\boldsymbol{\Lambda}^{(k)}\right) \boldsymbol{H}^{(k)}(u)+\mu u \boldsymbol{H}^{(k) \prime}(u)+\frac{\sigma^{2}}{2} u^{2} \boldsymbol{H}^{(k) \prime \prime}(u)+\boldsymbol{L}^{(k)} \boldsymbol{Q}_{2}^{(k)} \boldsymbol{g}^{(k)}(u)=\mathbf{0}, \quad u \in \mathcal{I}_{k}
$$

By solving $k$ independent differential equations (4.7), we obtain from Lemma 2.1 that

$$
H_{i}^{(k)}(u)=a_{i}^{(k)} u^{\eta_{i}^{(k)}}+b_{i}^{(k)} u^{\xi_{i}^{(k)}}+\frac{\theta_{i}^{(k)}}{\rho-\lambda_{i}^{(k)}-\mu} u-\frac{\zeta_{i}^{(k)}}{\rho-\lambda_{i}^{(k)}} I, \quad u \in \mathcal{I}_{k}, \quad i=1, \ldots, k
$$

where

$$
\begin{array}{ll}
\eta_{i}^{(k)}=f^{+}\left(\rho-\lambda_{i}^{(k)}\right)>1, & \xi_{i}^{(k)}=f^{-}\left(\rho-\lambda_{i}^{(k)}\right)<0 \\
\theta_{i}^{(k)}=\sum_{m=1}^{k} \ell_{i m}^{(k)} \sum_{j=k+1}^{N} q_{m j} s_{j}, & \zeta_{i}^{(k)}=\sum_{m=1}^{k} \ell_{i m}^{(k)} \sum_{j=k+1}^{N} q_{m j}
\end{array}
$$

and $a_{i}^{(k)}, b_{i}^{(k)}$ are unknown coefficients.

(iii) $u \in \mathcal{I}_{k^{*}}: \quad \mathcal{I}_{k^{*}}$ is a continuation region of $F_{1}(u), \ldots, F_{k^{*}}(u)$ while $F_{i}(u)=s_{i} u-I$ for $i=k^{*}+1, \ldots, N$. Thus, it is enough to consider the case $k^{*} \geq 1$. Since Bellman equation can be solved exactly in the same way as (ii) above, we obtain Equation (4.8). The difference is that, for $i=1, \ldots, k^{*}, H_{i}^{\left(k^{*}\right)}(u)$ must be asymptotically linear as $u \rightarrow \infty$ since $F_{i}(u) \approx G_{i k^{*}}(u)$ from Lemma 3.3. This implies the first term in Equation (4.8) must vanish and

$$
H_{i}^{\left(k^{*}\right)}(u)=b_{i}^{\left(k^{*}\right)} u^{\xi_{i}^{\left(k^{*}\right)}}+\frac{\theta_{i}^{\left(k^{*}\right)}}{\rho-\lambda_{i}^{\left(k^{*}\right)}-\mu} u-\frac{\zeta_{i}^{\left(k^{*}\right)}}{\rho-\lambda_{i}^{\left(k^{*}\right)}} I, \quad u \in \mathcal{I}_{k^{*}}, \quad i=1, \ldots, k^{*}
$$

So far, we have obtained the solutions (4.5), (4.8) and (4.9) of Bellman equation on all intervals $\mathcal{I}_{N}, \ldots, \mathcal{I}_{k^{*}}$. These solutions can be transformed into the value functions by $\boldsymbol{F}^{(k)}(u)=\boldsymbol{R}^{(k)} \boldsymbol{H}^{(k)}(u)$, or equivalently

$$
F_{i}^{(k)}(u)=\sum_{j=1}^{k} r_{j i}^{(k)} H_{j}^{(k)}(u), \quad u \in \mathcal{I}_{k}, \quad i=1, \ldots, k
$$


The number of unknown coefficients contained in Equation (4.10) is $\left(N-k^{*}\right)\left(N+k^{*}\right)$. To determine these coefficients as well as $\left(N-k^{*}\right)$ unknown thresholds $v_{k^{*}+1}, \ldots, v_{N}$, we invoke the value matching and smooth pasting conditions. These conditions are known to be a powerful tool to solve free boundary problems and widely used in literatures $[2,3,5,6,13]$.

Specifically, the value matching and smooth pasting conditions for $F_{i}(u)$ at $v_{k}$ are given as

$$
\lim _{u \uparrow v_{k}} F_{i}^{(k)}(u)=\lim _{u \downarrow v_{k}} F_{i}^{(k-1)}(u), \quad \lim _{u \uparrow v_{k}} F_{i}^{(k) \prime}(u)=\lim _{u \downarrow v_{k}} F_{i}^{(k-1) \prime}(u) .
$$

From Equation (4.10), these conditions can be rewritten in terms of $H_{i}^{(k)}(u)$ as

$$
\sum_{j=1}^{k} r_{j i}^{(k)} H_{j}^{(k)}\left(v_{k}\right)= \begin{cases}\sum_{j=1}^{k-1} r_{j i}^{(k-1)} H_{j}^{(k-1)}\left(v_{k}\right), & i=1, \ldots, k-1, \\ s_{k} v_{k}-I, & i=k\end{cases}
$$

and

$$
\sum_{j=1}^{k} r_{j i}^{(k)} H_{j}^{(k) \prime}\left(v_{k}\right)= \begin{cases}\sum_{j=1}^{k-1} r_{j i}^{(k-1)} H_{j}^{(k-1) \prime}\left(v_{k}\right), & i=1, \ldots, k-1, \\ s_{k}, & i=k\end{cases}
$$

for $k=k^{*}+1, \ldots, N$. We obtain $\left(N+k^{*}+1\right)\left(N-k^{*}\right)$ equations to determine the same number of unknowns. In general, the procedure to solve this system of nonlinear equations is implemented by numerical computation since they do not have explicit solutions except for 2-state case as we will show in the next section.

Remark 4.1 When $v_{N}<\cdots<v_{k^{*}+1}<\infty$ and $v_{k^{*}}=\cdots=v_{1}=\infty, F_{i}(u) \approx G_{i k^{*}}(u)$ for $i=1, \ldots, k^{*}$ from Lemma 3.3. To satisfy this asymptotic, the linear term of $F_{i}(u)$ in Equation (4.10) for $u \in \mathcal{I}_{k^{*}}$ must coincide with $g_{i}^{\left(k^{*}\right)}(\rho-\mu) u-h_{i}^{\left(k^{*}\right)}(\rho) I$. Specifically, Equation (4.10) is reduced to

$$
F_{i}(u)=\sum_{j=1}^{k^{*}} r_{j i}^{\left(k^{*}\right)} b_{j}^{\left(k^{*}\right)} u^{\xi_{j}^{\left(k^{*}\right)}}+g_{i}^{\left(k^{*}\right)}(\rho-\mu) u-h_{i}^{\left(k^{*}\right)}(\rho) I, \quad u \in \mathcal{I}_{k^{*}}, \quad i=1, \ldots, k^{*} .
$$

Equation (4.14) can also be verified directly from Equations (3.19) and (4.9).

Remark 4.2 In general, the solution of Bellman equation provides a candidate of the value function. To ensure that it is indeed the optimal value function, we will use the verification theorem which gives a sufficient condition for optimality. Specifically, if we can show

$$
\lim _{t \rightarrow \infty} e^{-\rho t} E_{i, u}\left[F_{S_{t}}\left(U_{t}\right)\right]=0
$$

for any initial states $i$ and $u$, then the above solutions of Bellman equation are the value functions. For verification theorem of the optimal stopping problems, see, for example, Chang [1] and Fleming and Soner [4].

To check Equation (4.15), we note from Lemma 3.3 and Equation (3.17) that there exists $u^{*}$ satisfying $F_{N}(u)>F_{k}(u)$ for $\forall u>u^{*}$ and $\forall k=1, \ldots, N-1$. Since $F_{N}(u)=s_{N} u-I$ for $u>v_{N}$,

$$
\begin{aligned}
E_{i, u}\left[F_{S_{t}}\left(U_{t}\right)\right] & =E_{i, u}\left[F_{S_{t}}\left(U_{t}\right) 1_{\left\{U_{t} \leq u^{*}\right\}}\right]+E_{i, u}\left[F_{S_{t}}\left(U_{t}\right) 1_{\left\{U_{t}>u^{*}\right\}}\right] \\
& \leq E_{i, u}\left[F_{S_{t}}\left(u^{*}\right)\right]+E_{i, u}\left[s_{N} U_{t}\right] \\
& =E_{i, u}\left[F_{S_{t}}\left(u^{*}\right)\right]+s_{N} u e^{\mu t}
\end{aligned}
$$

Since $E_{i, u}\left[F_{S_{t}}\left(u^{*}\right)\right]$ is bounded in $t$ and $\rho>\mu$, we obtain Equation (4.15). 


\section{Computational Issues and Numerical Examples}

In this section, we consider some computational issues for numerically obtaining the optimal policy and the value functions.

\subsection{Explicit solutions for 2-state case}

First, we solve the optimal investment problem explicitly when $\left\{S_{t}\right\}$ is a 2-state Markov process on $\mathcal{S}=\left\{s_{1}, s_{2}\right\}\left(s_{1}<s_{2}\right)$ governed by

$$
\boldsymbol{Q}=\left(\begin{array}{cc}
-\alpha & \alpha \\
\beta & -\beta
\end{array}\right)
$$

$\boldsymbol{Q}$ is diagonalizable in the following way:

$$
\boldsymbol{L}^{(2)} \boldsymbol{Q} \boldsymbol{R}^{(2)}=\left(\begin{array}{cc}
\frac{\beta}{\alpha+\beta} & \frac{\alpha}{\alpha+\beta} \\
-\frac{1}{\alpha+\beta} & \frac{1}{\alpha+\beta}
\end{array}\right)\left(\begin{array}{cc}
-\alpha & \alpha \\
\beta & -\beta
\end{array}\right)\left(\begin{array}{cc}
1 & -\alpha \\
1 & \beta
\end{array}\right)=\left(\begin{array}{cc}
0 & 0 \\
0 & -(\alpha+\beta)
\end{array}\right)=\boldsymbol{\Lambda}^{(2)} .
$$

From Lemma 3.2, $v_{2}<v_{1}$ always holds in this case. Since it is easy to calculate $f_{12}(s)=\alpha /(s+\alpha), k^{*}$ in Equation (3.20) is given as

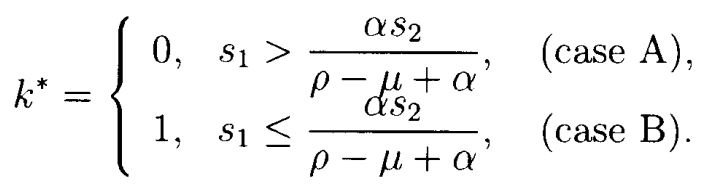

Since $v_{1}<\infty$ in case $\mathrm{A}$ while $v_{1}=\infty$ in case $\mathrm{B}$, the optimal policy in each case is given as follows:

\begin{tabular}{cccc}
\multicolumn{4}{c}{ case A } \\
\hline$S_{t}$ & $0<u<v_{2}$ & $v_{2} \leq u<v_{1}$ & $v_{1} \leq u$ \\
\hline$s_{1}$ & wait & wait & invest \\
\hline$s_{2}$ & wait & invest & invest \\
\hline
\end{tabular}

\begin{tabular}{ccc}
\multicolumn{3}{c}{ case B } \\
\hline$S_{t}$ & $0<u<v_{2}$ & $v_{2} \leq u$ \\
\hline$s_{1}$ & wait & wait \\
\hline$s_{2}$ & wait & invest \\
\hline
\end{tabular}

We will solve Bellman equation on $\mathcal{I}_{2}$ and $\mathcal{I}_{1}$ in this order.

(i) $u \in \mathcal{I}_{2}$ : From Equation (5.1), $\lambda_{1}^{(2)}=0, \lambda_{2}^{(2)}=-(\alpha+\beta), \boldsymbol{r}_{1}^{(2)}=(1,1)^{\top}$ and $\boldsymbol{r}_{2}^{(2)}=$ $(-\alpha, \beta)^{\top}$. We obtain from Equations (4.5) and (4.10) that

$$
\begin{array}{ll}
F_{1}(u)=F_{1}^{(2)}(u) \stackrel{\text { def }}{=} a_{1}^{(2)} u^{\eta_{1}^{(2)}}-\alpha a_{2}^{(2)} u^{\eta_{2}^{(2)}}, & 0<u<v_{2}, \\
F_{2}(u)=F_{2}^{(2)}(u) \stackrel{\text { def }}{=} a_{1}^{(2)} u^{\eta_{1}^{(2)}}+\beta a_{2}^{(2)} u^{\eta_{2}^{(2)}}, & 0<u<v_{2}
\end{array}
$$

where

$$
\eta_{1}^{(2)}=f^{+}(\rho)>1, \quad \eta_{2}^{(2)}=f^{+}(\rho+\alpha+\beta)>1 .
$$

(ii) $u \in \mathcal{I}_{1}: \mathcal{I}_{1}=\left(v_{2}, v_{1}\right)$ in case $\mathrm{A}$ and $\mathcal{I}_{1}=\left(v_{2}, \infty\right)$ in case B. In both cases, $F_{2}(u)=s_{2} u-I$ for $u>v_{2}$ and it suffices to consider $F_{1}(u)$. Since $\boldsymbol{Q}_{1}^{(1)}=(-\alpha)$, we see $\lambda_{1}^{(1)}=-\alpha$ and $\boldsymbol{r}_{1}^{(1)}=\boldsymbol{\ell}_{1}^{(1)}=(1)$ which implies $F_{1}(u)=H_{1}^{(1)}(u)$.

In case A, we obtain from Equation (4.8) and Remark 4.1 that

$$
F_{1}(u)=F_{1}^{(1 A)}(u) \stackrel{\text { def }}{=} a_{1}^{(1)} u^{\eta_{1}^{(1)}}+b_{1}^{(1)} u^{\xi_{1}^{(1)}}+\frac{\alpha s_{2}}{\rho+\alpha-\mu} u-\frac{\alpha}{\rho+\alpha} I, \quad v_{2}<u<v_{1}
$$

where $\eta_{1}^{(1)}=f^{+}(\rho+\alpha)$ and $\xi_{1}^{(1)}=f^{-}(\rho+\alpha)$. Note that $F_{1}(u)=r_{1} u-I$ for $u \geq v_{1}$ in case A. 
On the other hand in case B, we obtain from Equation (4.9) that

$$
F_{1}(u)=F_{1}^{(1 B)}(u) \stackrel{\text { def }}{=} b_{1}^{(1)} u^{\xi_{1}^{(1)}}+\frac{\alpha s_{2}}{\rho+\alpha-\mu} u-\frac{\alpha}{\rho+\alpha} I, \quad u>v_{2} .
$$

The unknown coefficients and thresholds should be determined by solving Equations (5.3)-(5.6) under the value matching and smooth pasting conditions. In what follows, we consider cases A and B separately.

Case A: In case A, there are 4 coefficients $a_{1}^{(2)}, a_{2}^{(2)}, a_{1}^{(1)}, b_{1}^{(1)}$ and 2 thresholds $v_{1}, v_{2}$ to be determined by

$$
\begin{aligned}
& F_{1}^{(2)}\left(v_{2}\right)=F_{1}^{(1 A)}\left(v_{2}\right), \quad F_{1}^{(1 A)}\left(v_{1}\right)=s_{1} v_{1}-I, \\
& F_{2}^{(2)}\left(v_{2}\right)=s_{2} v_{2}-I, \\
& F_{1}^{(2) \prime}\left(v_{2}\right)=F_{1}^{(1 A) \prime}\left(v_{2}\right), \quad F_{1}^{(1 A) \prime}\left(v_{1}\right)=s_{1}, \\
& F_{2}^{(2) \prime}\left(v_{2}\right)=s_{2} .
\end{aligned}
$$

From Equations (5.8) and (5.10), we can derive

$$
\left[\begin{array}{c}
a_{1}^{(1)} \\
b_{1}^{(1)}
\end{array}\right]=\frac{1}{\left(\eta_{1}^{(1)}-\xi_{1}^{(1)}\right) v_{1}^{\eta_{1}^{(1)}+\xi_{1}^{(1)}-1}}\left[\begin{array}{c}
d_{11} v_{1}^{\xi_{1}^{(1)}}+d_{12} v_{1}^{\xi_{1}^{(1)}-1} \\
d_{21} v_{1}^{\eta_{1}^{(1)}}+d_{22} v_{1}^{\eta_{1}^{(1)}-1}
\end{array}\right]
$$

where

$$
\begin{array}{ll}
d_{11}=\left(\xi_{1}^{(1)}-1\right)\left(\frac{\alpha s_{2}}{\rho+\alpha-\mu}-s_{1}\right), & d_{12}=\xi_{1}^{(1)} \frac{\rho I}{\rho+\alpha} \\
d_{21}=\left(\eta_{1}^{(1)}-1\right)\left(s_{1}-\frac{\alpha s_{2}}{\rho+\alpha-\mu}\right), & d_{22}=-\eta_{1}^{(1)} \frac{\rho I}{\rho+\alpha} .
\end{array}
$$

We also derive from Equations (5.7) and (5.9)

$$
\left[\begin{array}{l}
a_{1}^{(2)} \\
a_{2}^{(2)}
\end{array}\right]=\frac{1}{\beta\left(\eta_{2}^{(2)}-\eta_{1}^{(1)}\right) v_{2}^{\eta_{1}^{(1)}+\eta_{2}^{(2)}-1}}\left[\begin{array}{c}
\beta\left(\eta_{2}^{(2)}-1\right) v_{2}^{\eta_{2}^{(2)}} s_{2}-\beta \eta_{2}^{(2)} v_{2}^{\eta_{2}^{(2)}-1} I \\
-\left(\eta_{1}^{(2)}-1\right) v_{2}^{\eta_{1}^{(2)}} s_{2}+\eta_{1}^{(2)} v_{2}^{\eta_{1}^{(2)}-1} I
\end{array}\right] .
$$

Combining Equation (5.12) with Equations (5.7) and (5.9), we can further show

$$
\left[\begin{array}{l}
a_{1}^{(1)} \\
b_{1}^{(1)}
\end{array}\right]=\frac{1}{\left(\eta_{1}^{(1)}-\xi_{1}^{(1)}\right) v_{2}^{\eta_{1}^{(1)}+\xi_{1}^{(1)}-1}}\left[\begin{array}{c}
\left(e_{21}-\xi_{1}^{(1)} e_{11}\right) v_{2}^{\xi_{1}^{(1)}}+\left(e_{22}-\xi_{1}^{(1)} e_{12}\right) v_{2}^{\xi_{1}^{(1)}-1} \\
\left(\eta_{1}^{(1)} e_{11}-e_{21}\right) v_{2}^{\eta_{1}^{(1)}}+\left(\eta_{1}^{(1)} e_{12}-e_{22}\right) v_{2}^{\eta_{1}^{(1)}-1}
\end{array}\right]
$$

where

$$
\begin{aligned}
& e_{11}=\left\{\frac{\beta\left(\eta_{2}^{(2)}-1\right)+\alpha\left(\eta_{1}^{(2)}-1\right)}{\beta\left(\eta_{2}^{(2)}-\eta_{1}^{(2)}\right)}-\frac{\alpha}{\rho+\alpha-\mu}\right\} s_{2}, \\
& e_{12}=\left\{\frac{\alpha}{\rho+\alpha}-\frac{\beta \eta_{2}^{(2)}+\alpha \eta_{1}^{(2)}}{\beta\left(\eta_{2}^{(2)}-\eta_{1}^{(2)}\right)}\right\} I, \\
& e_{21}=\left\{\frac{\beta \eta_{1}^{(2)}\left(\eta_{2}^{(2)}-1\right)+\alpha \eta_{2}^{(2)}\left(\eta_{1}^{(2)}-1\right)}{\beta\left(\eta_{2}^{(2)}-\eta_{1}^{(2)}\right)}-\frac{\alpha}{\rho+\alpha-\mu}\right\} s_{2}, \\
& e_{22}=-\frac{(\alpha+\beta) \eta_{1}^{(2)} \eta_{2}^{(2)}}{\beta\left(\eta_{2}^{(2)}-\eta_{1}^{(2)}\right)} I
\end{aligned}
$$


Now let $x=v_{1} / v_{2}$, then $x>1$ from Lemma 3.2. We substitute $v_{1}=x v_{2}$ into Equation (5.11) and equate it to Equation (5.13). With some algebras, we can show

$$
v_{2}=\frac{d_{12}-\left(e_{22}-\xi_{1}^{(1)} e_{12}\right) x^{\eta_{1}^{(1)}}}{\left(e_{21}-\xi_{1}^{(1)} e_{11}\right) x^{\eta_{1}^{(1)}}-d_{11} x}=\frac{d_{22}+\left(e_{22}-\eta_{1}^{(1)} e_{12}\right) x^{\xi_{1}^{(1)}}}{\left(\eta_{1}^{(1)} e_{11}-e_{21}\right) x^{\xi_{1}^{(1)}}-d_{21} x} .
$$

Since $d_{i j}$ 's and $e_{i j}$ 's are known coefficients, the second equality of Equation (5.14) is a nonlinear equation of a single variable $x$ which can easily be solved numerically. Once $x$ is at hand, we can calculate $v_{2}$ from Equation (5.14), $v_{1}=k v_{2}, a_{1}^{(2)}$ and $a_{2}^{(2)}$ from Equation (5.12), $a_{1}^{(1)}$ and $b_{1}^{(1)}$ from Equation (5.11).

Case B: In case B, we need to determine 4 unknowns $a_{1}^{(2)}, a_{2}^{(2)}, b_{1}^{(1)}$ and $v_{2}$ from

$$
\begin{aligned}
& F_{1}^{(2)}\left(v_{2}\right)=F_{1}^{(1 B)}\left(v_{2}\right), \quad F_{2}^{(2)}\left(v_{2}\right)=s_{2} v_{2}-I, \\
& F_{1}^{(2) \prime}\left(v_{2}\right)=F_{1}^{(1 B)}\left(v_{2}\right), \quad F_{2}^{(2) \prime}\left(v_{2}\right)=s_{2}
\end{aligned}
$$

which can be solved explicitly. Specifically, we obtain after some algebras

$$
v_{2}=\frac{(\rho+\alpha-\mu)\left\{\left(\xi_{1}^{(1)}-\eta_{1}^{(2)}\right) \beta \rho \eta_{2}^{(2)}+\left(\xi_{1}^{(1)}-\eta_{2}^{(2)}\right)(\rho+\alpha+\beta) \alpha \eta_{1}^{(2)}\right\} I}{(\rho+\alpha)\left\{\left(\xi_{1}^{(1)}-\eta_{2}^{(2)}\right)\left(\eta_{1}^{(2)}-1\right)(\rho+\alpha+\beta-\mu) \alpha+\left(\xi_{1}^{(1)}-\eta_{1}^{(2)}\right)(\rho-\mu)\left(\eta_{2}^{(2)}-1\right) \beta\right\} s_{2}}
$$

and

$$
\begin{aligned}
a_{1}^{(2)} & =\frac{\left(\eta_{2}^{(2)}-1\right) \beta v_{2} s_{2}-\eta_{2}^{(2)} \beta I}{\left(\eta_{2}^{(2)}-\eta_{1}^{(2)}\right) \beta v_{2}^{\eta_{1}^{(2)}}}, \\
a_{2}^{(2)} & =\frac{\left(1-\eta_{1}^{(2)}\right) v_{2} s_{2}+\eta_{1}^{(2)} I}{\left(\eta_{2}^{(2)}-\eta_{1}^{(2)}\right) \beta v_{2}^{\eta_{2}^{(2)}}} \\
b_{1}^{(1)} & =\frac{\left(\eta_{1}^{(2)}-1\right)\left(1+\frac{\beta}{\rho+\alpha-\mu}\right) \alpha s_{2} v_{2}-\left(1+\frac{\beta}{\rho+\alpha}\right) \alpha \eta_{1}^{(2)} I}{\left(\xi_{1}^{(1)}-\eta_{1}^{(2)}\right) \beta v_{2}^{\xi_{1}^{(1)}}} .
\end{aligned}
$$

\subsection{Numerical procedure for multi-state case}

Since we do not expect to get explicit solutions when there are multiple states, we instead propose a simple numerical procedure for computing the optimal policy and the value functions.

As stated in Section 4, there are $N-k^{*}$ threshold values and $\left(N+k^{*}\right)\left(N-k^{*}\right)$ unknown coefficients of the value functions to be determined by the same number of nonlinear equations (4.12) and (4.13). A key observation is that Equations (4.12) and (4.13) are system of linear equations in terms of unknown coefficients if the threshold values $v_{k^{*}+1}, \ldots, v_{N}$ are given. Thus, we can construct the following iterative procedure.

1. Set initial trial values of $v_{k^{*}+1}, \ldots, v_{N}$.

2. Compute unknown coefficients by solving $\left(N+k^{*}\right)\left(N-k^{*}\right)$ equations out of $\left(N+k^{*}+\right.$ 1) $\left(N-k^{*}\right)$ equations contained in (4.12) and (4.13).

3 . If the coefficients obtained in Step 2 satisfy the remaining $N-k^{*}$ equations in (4.12) and (4.13) within prescribed computational error, then we get the solutions.

Otherwise, we set new trial values of $v_{k^{*}+1}, \ldots, v_{N}$ and Goto Step 2.

This procedure reduces the original problem with $\left(N+k^{*}+1\right)\left(N-k^{*}\right)$ unknowns to much simpler problem with $N-k^{*}$ unknowns and performs quite well as far as we compute the numerical results given in the next subsection. 


\subsection{Numerical examples}

In this subsection, we show some numerical results. Table 1 summarizes the parameters of the base model for 2-state case. Roughly speaking, an annual growth rate of the project

Table 1: Parameters for the base case of numerical examples (2-state case)

\begin{tabular}{cccccccc}
\hline$\mu$ & $\sigma$ & $\rho$ & $q_{12}$ & $q_{21}$ & $s_{1}$ & $s_{2}$ & $I$ \\
\hline 0.05 & 0.1 & 0.1 & 0.1 & 0.1 & 1 & 1.4 & 1 \\
\hline
\end{tabular}

value is $5 \%$ with volatility $10 \%$, and future payoff will be discounted $10 \%$ per year. The Markov process changes its state once a 10 year in average.

Table 2 shows how the ratio $s_{2} / s_{1}$ affects the thresholds (we fix $s_{1}=1$ ). For the above parameters, case A or B in Equation (5.2) occurs according to $s_{2}<1.5$ or $s_{2} \geq 1.5$, respectively. In this case, an investment will be executed only when $S_{t}=s_{2} \cdot v_{2}$ decreases as $s_{2}$ increases due to stronger incentive to invest before the Markov process moves to $s_{1}$. In fact, the payoff obtained by investing at $v_{2}$ is around 1.95 for all values of $s_{2}$ listed in Table 2. Figure 1 shows the value functions $F_{1}(u)$ and $F_{2}(u)$ for $s_{2}=1.2$ and $s_{2}=2.0$,

Table 2: $s_{2}$ and threshold values $v_{1}$ and $v_{2}\left(s_{1}=1\right)$

\begin{tabular}{cccccc}
\hline$s_{2}$ & 1.2 & 1.4 & 1.6 & 1.8 & 2.0 \\
\hline$v_{1}$ & 3.61 & 10.8 & $\infty$ & $\infty$ & $\infty$ \\
$v_{2}$ & 1.64 & 1.40 & 1.22 & 1.08 & 0.97 \\
\hline
\end{tabular}

respectively. Each curve is composed of smoothly pasted 2 or 3 different functions.
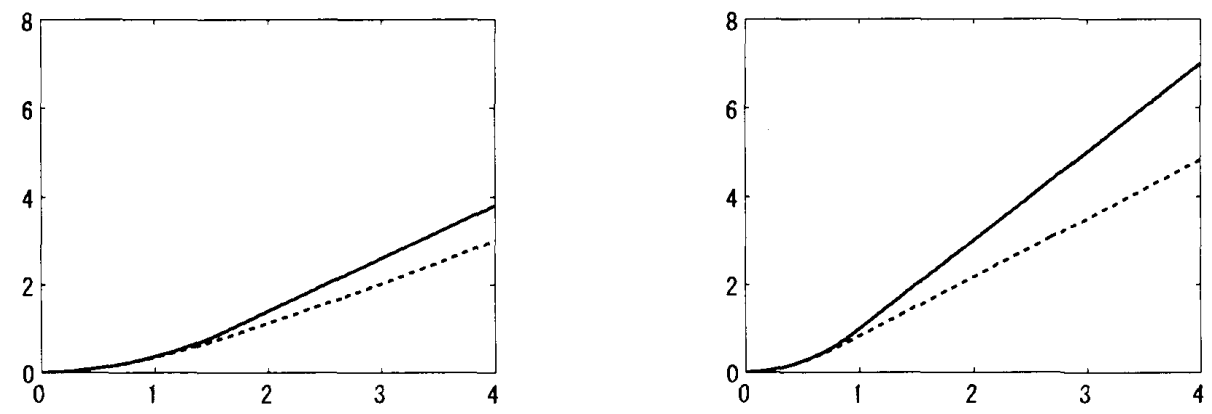

Figure 1: Value functions $F_{1}(u)$ (dashed line) and $F_{2}(u)$ (solid line) for $s_{2}=1.2$ (left panel) and $s_{2}=2.0$ (right panel). Other parameters are the same as in Table 1

Table 3 shows thresholds when transition rates $q_{12}$ and $q_{21}$ of the Markov process range from 0.1 to 1.0 (from 10 years to 1 year in average). $v_{2}$ increases as $q_{12}=q_{21}$ increases since the possibility of risk to stay in state $s_{1}$ for longer time decreases. However, the difference is rather small for these parameters since it is almost unlikely to occur to invest in state $s_{1}$.

For 3 -state case, we use the same parameters of $\mu, \sigma, \rho$ and $I$ as in Table 1 . The transition rate matrix is given by

$$
\boldsymbol{Q}=\left(\begin{array}{ccc}
-0.5 & 0.5 & 0 \\
0.2 & -0.4 & 0.2 \\
0 & 0.5 & -0.5
\end{array}\right)
$$


Table 3: Rate of regime transitions and threshold levels

\begin{tabular}{ccccc}
\hline$q_{12}=q_{21}$ & 0.1 & 0.2 & 0.5 & 1.0 \\
\hline$v_{1}$ & 10.8 & $\infty$ & $\infty$ & $\infty$ \\
$v_{2}$ & 1.40 & 1.43 & 1.48 & 1.51 \\
\hline
\end{tabular}

Since $\boldsymbol{Q}$ given in (5.15) satisfies the condition (3.7), we know $v_{1} \geq v_{2} \geq v_{3}$ in advance for $s_{1}<s_{2}<s_{3}$. Table 4 summarizes the results for $s_{2}=1.2,1.4,1.6$ with $s_{1}=1$ and $s_{3}=1.8$ being fixed. For all values of $s_{2}, v_{1}=\infty$ meaning that it is never optimal to invest when $S_{t}=s_{1}$ since $s_{1}$ is small compared with $s_{3} . v_{2}$ is also infinity when $s_{2}=1.2$ by the same reason while it becomes finite and decreases as $s_{2}$ increases because investment when $S_{t}=s_{2}$ becomes more attractive.

Table 4: $s_{2}$ and threshold values $v_{1}, v_{2}$ and $v_{3}\left(s_{1}=1, s_{3}=1.8\right)$

\begin{tabular}{cccc}
\hline$s_{2}$ & 1.2 & 1.4 & 1.6 \\
\hline$v_{1}$ & $\infty$ & $\infty$ & $\infty$ \\
$v_{2}$ & $\infty$ & 9.25 & 2.07 \\
$v_{3}$ & 1.01 & 1.01 & 1.02 \\
\hline
\end{tabular}

Finally, we will show an example in which all threshold values are finite. This case will occur if the difference between scale parameters are not large. Figure 2 shows the value functions for $s_{1}=1.00, s_{2}=1.05$ and $s_{3}=1.10$ with the other parameters being the same as above. The threshold values in this case are calculated as $v_{1}=4.24, v_{2}=2.32$ and $v_{3}=1.75$.

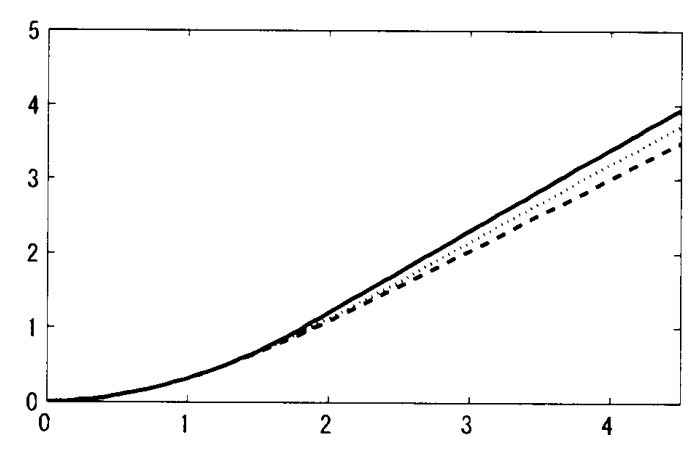

Figure 2: Value functions $F_{1}(u)$ (dashed line), $F_{2}(u)$ (dotted line) and $F_{3}(u)$ (solid line)

Acknowledgements The author is grateful to the editors and anonymous referees for helpful comments. Especially, a comment from one of the referees lead to an extension of the original problem. He also thanks Mitsuhiko Mori for valuable discussions.

\section{References}

[1] F.-R. Chang: Stochastic Optimization in Continuous Time (Cambridge University Press, 2004).

[2] J. Décamps, T. Mariotti and S. Villeneuve: Irreversible investment in alternative projects. Economic Theory, 28 (2006), 425-448. 
[3] A.V. Dixit and R.S. Pindyck: Investment under Uncertainty (Princeton University Press, 1994).

[4] W.H. Fleming and H.M. Soner: Controlled Markov Processes and Viscosity Solutions (Springer-Verlag, 1992).

[5] X. Guo: An explicit solution to an optimal stopping problem with regime switching. Journal of Applied Probability, 38 (2001), 464-481.

[6] X. Guo, J. Miao and E. Morellec: Irreversible investment with regime shifts. Journal of Economic Theory, 122 (2005), 37-59.

[7] M. Kijima: Markov Processes for Stochastic Modeling (Chapman \& Hall, 1997).

[8] M. Kijima: Stochastic Processes with Applications to Finance (Chapman \& Hall, 2003).

[9] M. Mori: Optimal investment with bivariate growth options and state dependent capacity. Master thesis, Graduate School of Business Sciences, University of Tsukuba (2006).

[10] M. Ohnishi and M. Tsujimura: An impulse control of a geometric Brownian motion with quadratic costs. European Journal of Operations Research, 168 (2006), 311-321.

[11] B. Oksendal: Stochastic Differential Equations, 5th ed. (Springer, 1998).

[12] E.S. Schwartz and L. Trigeorgis: Real Options and Investment under Uncertainty (MIT Press, 2004).

[13] A. Vollert: A Stochastic Control Framework for Real Option in Strategic Valuation (Birkhäuser, 2003).

Naoki Makimoto

Graduate School of Business Sciences

University of Tsukuba

3-29-1 Otsuka, Bunkyo

Tokyo 112-0012, Japan

E-mail: makimoto@gssm.gsbs.tsukuba.ac.jp 